\title{
Permanent pacemaker implantation in a challenging anatomy: Persistent left superior vena cava
}

\author{
Alexandru Bostan ${ }^{*}$, , Dora Diana Astratinei ${ }^{1}$, Narcis Tăbăcaru ${ }^{1}$, Ștefan Ailoaei ${ }^{1}$, \\ Cristian Stătescu ${ }^{1,2}$
}

1"Prof. Dr. George I.M. Georgescu" Cardiovascular Diseases Institute, Iasi; 2"Grigore T. Popa" University of Medicine and Pharmacy, Iasi, Romania

\begin{abstract}
The persistence of the left superior vena cava is one of the most common abnormalities that could affect the thoracic venous return, despite its rare occurrence. It can usually be found as the only or in combination with other congenital cardiac abnormalities. Even though it is usually asymptomatic and it rarely has important consequences on the hemodynamics, it could sometimes represent a serious threat. In this regard, PLSVC often represents an incidental finding during an invasive procedure or imaging. We present an interesting case of a 66-year-old patient, with permanent atrial fibrillation and chronic kidney disease who presented to our clinic for a syncope due to complete atrioventricular block. The implant procedure was marked by the incidental intraprocedural finding of unusual venous anatomy. This anomaly included the absence of the superior vena cava, with the communication of the right brachiocephalic trunk and right subclavian vein with a persistent left superior vena cava which drainage directly into the coronary sinus. The right-side approach, as well as the limitation of using contrast-based venography, due to the kidney disease, made the procedure more difficult, but the final position of an active fixation ventricular lead was successfully achieved with optimal and stable pacing parameters through the formation of a particular curve of the lead stylet. Persistence of the left superior vena cava is a venous anomaly, which is frequently suspicioned at transthoracic echocardiography examination when the examiner found a dilated coronary sinus but diagnosed on the implant table of a cardiac device. These anomalies can pose problems and exponentially increase the procedural time even in experienced hands.
\end{abstract}

Keywords: cardiac pacing, persistent left superior vena cava, coronary sinus implantation technique, venography.

\section{Introduction}

Rarely diagnosed, persistence of the left superior vena cava (PLSVC) is one of the most common congenital abnormalities of thoracic venous return and can be found in a percentage of $0.3-0.5 \%$ of individuals with a normal heart and up to $4.5 \%$ in those with congenital heart disease [1]. In $80-90 \%$ of the cases, the left

Received: February 2020; Accepted after review: August 2020; Published: August 2020.

*Corresponding author: Alexandru Bostan, Cardiology Department, "Prof. Dr. George I.M. Georgescu" Cardiovascular Diseases Institute, 50 Bvd. Carol I, 700503, lasi, Romania

Email: bostanpalexandru@yahoo.com superior vena cava may coexist with the superior vena cava and may be accompanied by other cardiac malformations such as dextrocardia, tetralogy Fallot, aortic coarctation, transposition of large vessels [2, 3]. Normally, the right internal jugular vein and the right subclavian vein unite to form the right brachiocephalic trunk, which by joining with left correspondent form the superior vena cava. In the case of the persistence of the left superior vena cava, the right brachiocephalic trunk joins the left subclavicular vein and the left internal jugular vein and drains through PLSVC with opening through the coronary sinus ostium in the right atrium. The coronary sinus can be found dilated at echo exam as the only sign of 
the presence of this anomaly. The literature also describes situations in which PLSVC joins the upper left pulmonary vein and opens in the left atrium, but these are very rare cases [4]. The prognosis of these patients depends on the associated malformations [5]. When is isolated, PLSVC does not generate significant hemodynamic impact, this abnormality of the vein system often remain asymptomatic and may complicate the implant procedures of cardiac devices, such as pacemakers, ICDs, or cardiac resynchronization therapy, as well as in obtaining a vascular approach like a dialysis catheter [6]. The literature is quite poor in cases of implantation of the transvenous pacing electrodes through a persistent left superior vena cava. What is really difficult is the positioning of the right ventricular electrode because of the sharp angles which the lead has to travel to the final position, either at the apical level or at the level of the interventricular septum [7-13]. Theoretically, those sharp angles of the ventricular lead seem to have an increased risk of lead fracture but we did not find any reported case of right ventricular lead fracture placed through a PLSVC. To cross the tricuspid valve by the right ventricular lead, we used a hand-prepared lead stylet with two particular curves. The primary curvature is wider, which by creating a large loop on the atrial free wall forced the electrode to prolapse in the right ventricle. Once we reached the right ventricle, the secondary curve, a small one, in the frontal plane, helps to position the lead on the interventricular septum.

\section{Case report}

A 66-year-old patient, symptomatic through dizziness and lipotimic events at home, is referred to our clinic by the cardiologist of a territorial hospital for permanent electrical cardio stimulation after the electrocardiogram revealed a severe conduction disorder. The patient's medical history was relevant for permanent atrial fibrillation, hypertension, type 2 diabetes complicated with nephropathy and retinopathy. The clinical examination was significant for bradycardic but rhythmic heart sounds. The pulse of the peripheral arteries was present. Laboratory tests shows creatinine $=3.2 \mathrm{mg} / \mathrm{dl}$, urea $=73 \mathrm{mg} / \mathrm{dl}$. GFR=20.8 ml/ $\mathrm{ml} / 1.73 \mathrm{~m}^{2}$ (CKD stage IV), $\mathrm{K}=5.9 \mathrm{mEq} / \mathrm{l}$, $I N R=2.1$, glucose $=156 \mathrm{mg} / \mathrm{dl}$. ECG reveals atrial fibrillation with complete atrioventricular block with junctional escape approx. 42bpm (Figure 1). Echocardiography revealed: normal sized left ventricle, mildly hypertrophied, with mild systolic dysfunction, without wall motion abnormalities. Slight dilation of AS. Absence of pericardial effusion and intracavitary formations as well as structural abnormalities. A dilated coronary sinus raised the suspicion of PLSVC (Figure 2).

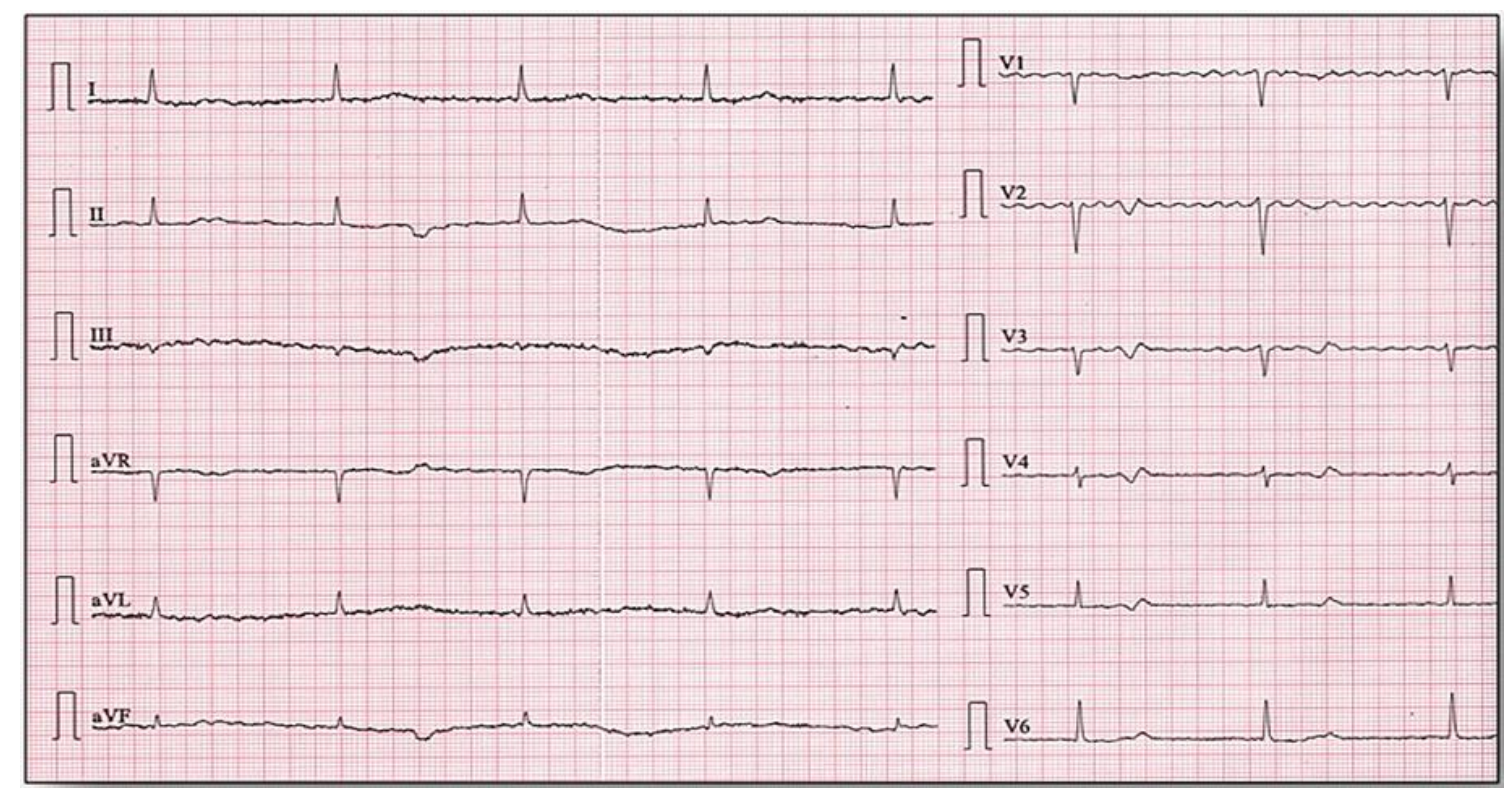

Fig. 1. ECG shows atrial fibrillation with complete atrioventricular block with an escape rhythm of $42 / \mathrm{min}$, narrow QRS and normal QRS axis (30 degrees). 

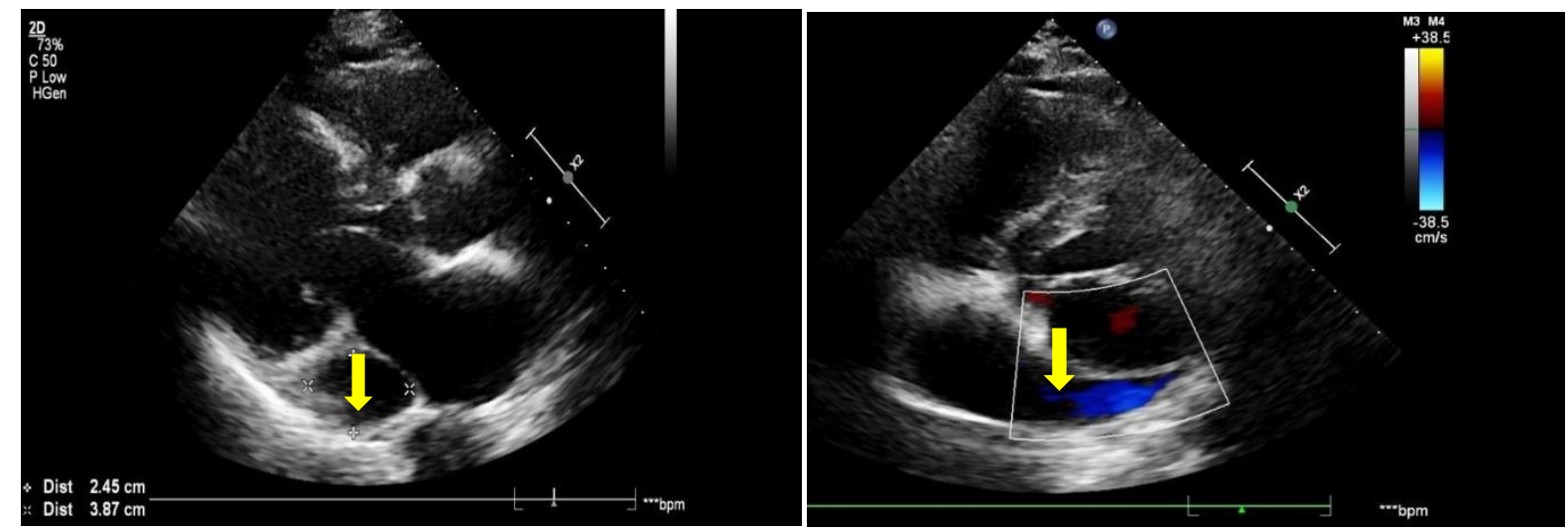

Fig. 2. Transthoracic echocardiogram: parasternal long axis $(A)$, and modified short axis view $(B)$ illustrating a dilated coronary sinus (yellow arrow)

Because the patient was left-handed and showed signs of an old left clavicle fracture, we opted for the right-side approach. After we find out the right cephalic vein and advancing the guidewire with an abnormal path along the left edge of the cardiac silhouette, and not on the right-side as we would have expected in the case of the normal superior vena cava (Figure $3)$.
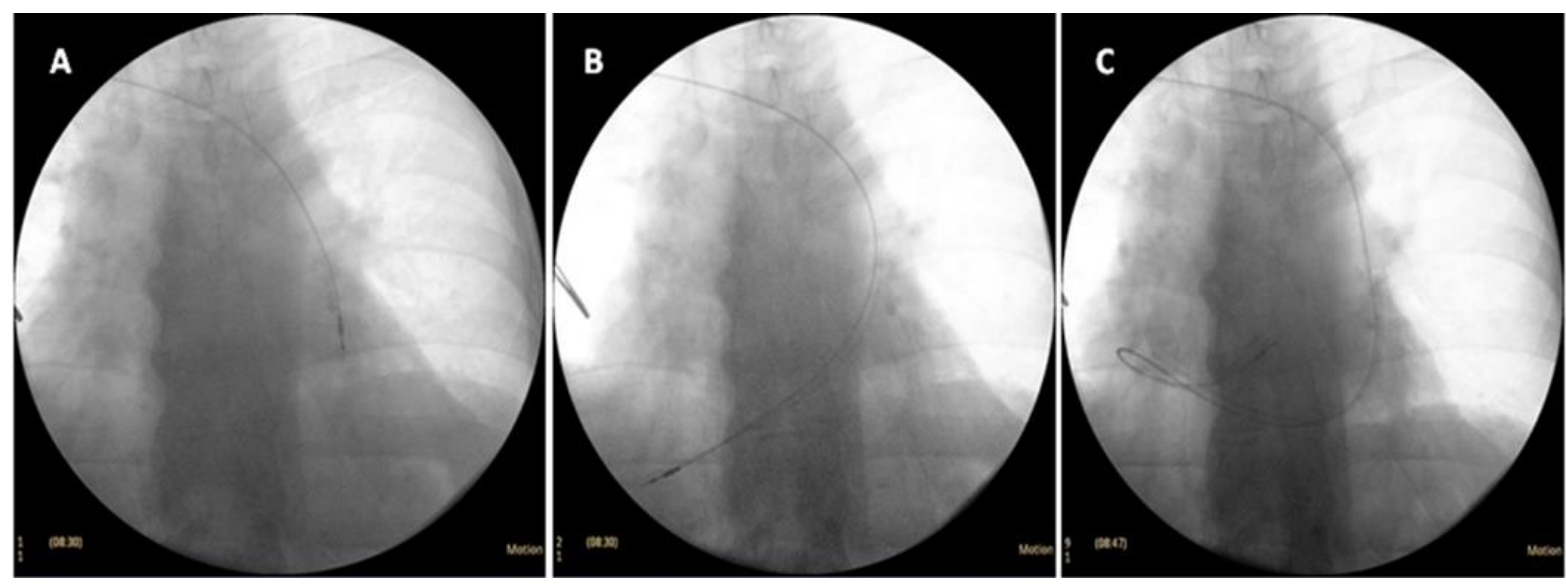

Fig. 3. A. After the lead introduction in the right subclavian vein, it takes an abnormal trajectory to the left side of the spine. B. The second image shows the point of the electrode exit through the coronary sinus in the right atrium. C. The third image shows the final position of the right ventricular lead on the interventricular septum after crossing the tricuspid annulus

We were placed in front of an absence of normal superior vena cava where brachiocephalic trunk and entire superior venous system drain through a persistent left superior vena cava. Constrained by the impaired renal function of the patient, we did not use contrast venography, a maneuver that would have been a real help for the detailed evaluation of the venous malformation. We initially tried to find a viable superior vena cava, which could accompany the persistent left superior vena cava, but our attempt was unsuccessful. Thus, the guide wire was advanced, reaching the level of the right atrium through the ostium of the coronary sinus. Since the coronary sinus opens very close to the tricuspid annulus, crossing the tricuspid valve in attempt to find a stable and optimal position of the ventricular lead it was a real challenge. Initially, we gave a smooth large curve (primary curve) of the stylet and additionally, a secondary curved in the frontal plane. Under fluoroscopy, with considerable procedural time, approximative 18 minutes (in patients with normal anatomy, mean fluoroscopy time in our lab for a VVI pacemaker implantation is no more than 2.5 minutes), it was possible to cross the tricuspid annulus and place a $58 \mathrm{~cm}$ bipolar 
lead of pace and sense with active fixation at the interventricular septum (Figure 3) with very good pacing parameters: RV sensing $13 \mathrm{mV}$, impedance - $690 \mathrm{Ohm}$, pacing threshold $0.5 \mathrm{~V}$, with a duration of the stimulus of $0.4 \mathrm{~ms}$.

\section{Discussion}

In this case report we want to describe our technique of implanting a unicameral transvenous pacemaker in a patient with persistent left superior vena cava diagnosed during the implant. During the procedure of implanting a cardiac device, the persistence of the left superior vena cava causes an increased difficulty when attempting the approach on the left-side, and it becomes even more difficult when attempting the approach on the right-side due to the narrow angles that appear along the path of the venous system [14]. A PLSVC may coexist with other congenital heart defects and early detection may be particularly important for the patient's prognosis. Isolated PLSVC without coexisting with a superior vena cava is very rare, a reported percentage of about $0.1 \%$. Given the chronic kidney disease in the predialysis stage that the patient presented, we have decided to refrain from using contrast venography to objectify an eventual superior cava vein. In approximately $10 \%$ of cases, persistent left superior vena cava can drain into the left atrium causing significant hemodynamic effects [15-17]. Possible preprocedural clues of abnormal venous drainage could be seen on the chest $\mathrm{x}$-ray postero-anterior incidence that may show enlargement of the aortic shadow, or left paramediastinal bulging [18]. The most important sign of PLSVC is provided by transthoracic echocardiography. It consists of a finding of a dilated coronary sinus. As we found at our patient (Figure 2), the echo exam raised the suspicion of a PLSVC, but the history of a clavicle fracture on the left side, that could make very difficult the vascular access forced us to use the right-sided approach. A standard implant technique of a cardiac device used in a patient with significant venous return abnormalities cannot be applied to all patients with PLSVC due to the unique anatomical condition of each patient. The transthoracic echocardiogram plays a crucial role in the evaluation of these patients before invasive cardiac procedures. Structures like the Chiari network or Eustachian valve, which are results of incomplete resorption of the sinus venosus during embryonic development often without significant clinical relevance can constitute a real barrier in the insertion of catheters or pacemaker leads [19]. Knowing those abnormalities before the time of the procedures by performing a proper echo assessment can change the strategy in approaching these kinds of patients. Previously suspected but undiagnosed, an implant table venography would have clarified the particular anatomy of this patient, but limited by the chronic kidney disease, as well as successfully positioning the right ventricular stimulation electrode with optimal stimulation parameters, was the right method for this patient. In the situation where an acceptable positioning of the right ventricular electrode would not have been achieved, in extremis, venography with contrast substance would have been used to clarify the particular anatomical condition [14]. To maintain a stable position of the right ventricular electrode, subjected to an important tension due to the narrow angles that they cross in the path to the right ventricle, we used an active fixation lead. The results of a recently published study demonstrate the clinical safety of implanting the permanent transvenous pacemaker by using the active fixation right ventricle electrodes [20]. In a recently published case report, Sasaki et al. describes the useful technique of implanting the ventricular electrode through a fixed sheath with which they cannulated the coronary sinus by using the left-side approach, which would have been difficult on the right-side because of the narrow angle between the right brachiocephalic system and the persistent left superior vena cava [21].

\section{Conclusion}

Particular anatomical situations such as persistence of the left superior vena cava, often asymptomatic, may occur during the implantation procedure of a cardiac device, a real challenge for the implanters. Contrast substance venography clarifies venous abnormalities and may be particularly useful in 
the case of a patient with venous abnormalities, but patients should be carefully selected given the increased risk of contrast nephropathy.

We presented the particular case of a patient whose pacemaker VVIR implant was a real challenge after the intraprocedural diagnosis of PLSVC, without being able to use contrast substance venography due to the underlying renal impairment, but it was possible to obtain an optimal position of the stimulation electrode at the level of the interventricular septum. An adequate echographic examination is mandatory before cardiac pacemaker implantation. A suspicion of PLSVC could be clarified using transthoracic saline contrastenhanced echocardiography. There are other abnormal structures as Chiari network, Eustachian valve which are serious concerns

\section{References}

1. Zhong $Y L$, Long $X-M$, Jiang $L Y$, et al. Surgical treatment of dextroversion, isolated persistent left superior vena cava draining into the left atrium. J Card Surg 2015; 30(10):767-770.

2. Ruano CA, Marinho-da-Silva $A$, Donato $P$. Congenital thoracic venous anomalies in adults: morphologic MR imaging. Curr Probl Diagn Radiol 2015; 44(4):337-345.

3. Kula S, Cevik A, Sanli C, et al. Persistent left superior vena cava: experience of a tertiary health-care center. Pediatr Int 2011; 53(6):1066-1069.

4. Maki R, Miyajima M, Mishina $T$, Watanabe A. Left upper pulmonary vein connected to the persistent left superior vena cava and the left atrium. Gen Thorac Cardiovasc Surg 2019; 67(8):723-725.

5. Rao EMM, Paydak H, Mehta J. Right Sided Approach for a Pacemaker Insertion in the presence of Persistent Left Sided Superior Vena Cava: A Pacing Challenge. Clin Med Rev Case Rep 2015; 2:31-32.

6. He H, Li B, Ma Y, Zhang Y, Ye C, Mei C, Liu Y. Catheterization in a patient with end-stage renal disease through persistent left superior vena cava: a rare case report and literature review. BMC Nephrol 2019; 20(1):200-202.

7. Feldman A, Antonelli D, Turgeman $\mathrm{Y}$. Successful right ventricular lead placement using a right ventricular septal stylet in a patient with persistent left superior vena cava. Europace 2013; 15:354-354.

8. Roka A, Merkely B. Dual-chamber pacemaker implantation via both superior vena cavae in a and can increase the complexity of procedures. Knowing these anomalies, especially when the electrode takes an unexpected trajectory, can decrease the implanter's anxiety level, allowing the use of new approaches to successfully perform the procedure.

\section{Consent}

Written informed consent was obtained from the patient for publication of this case report and the accompanying images. A copy of the written consent is available for review by the Editor-in-Chief of this journal.

\section{Competing interests}

The authors declare that they have no competing interests. patient with persistent left superior vena cava. Heart Rhythm 2011; 8:1815-1816.

9. Umar F, Alzuwam A, Osman F. Dual-chamber pacemaker in persistent left superior vena cava. Heart 2011; 97:1360-1361.

10. Porcellini S, Rimini A, Biasi S. Pacemaker implantation in a patient with persistent left superior vena cava using a steerable catheterdelivered lead. J Cardiovasc Med 2012; 13:653655.

11. Pontillo D, Patruno N. Persistent left superior vena cava and pacemaker implantation. World J Cardiol 2013; 5:373-374.

12. Dabrowski $P$, Obszanski $B$, Kleinrok $A$, Kutarski A. Long-term follow-up after pacemaker implantation via persistent left superior vena cava. Cardiol J 2014; 21:413-418.

13. Hassine M, Hamdi S, Chniti G, Boussaada M, Bouchehda N, Mahjoub M. Permanent cardiac pacing in a patient with persistent left superior vena cava and concomitant agenesis of the right-sided superior vena cava. J Arrhythm 2015; 31:326-327.

14. Rizkallah J, Burgess J, Kuriachan V. Absent right and persistent left superior vena cava: troubleshooting during a challenging pacemaker implant:a case report. BMC Res Notes 2014; $7(1): 1-4$.

15. Klimek-Piotrowska W, Hołda MK, Piątek K, Koziej M, Hołda J. Normal distal pulmonary vein anatomy. Peer J 2016; 4:1579-1579.

16. Elison B, Evans D, Zanders T, Jeanmonod R. Persistent left superior vena cava draining into the pulmonary venous system discovered after 
central venous catheter placement. Am J Emerg Med 2014; 32(8):943-943.

17. Povoski SP, Khabiri H. Persistent left superior vena cava: review of the literature, clinical implications, and relevance of alterations in thoracic central venous anatomy as pertaining to the general principles of central venous access device placement and venography in cancer patients. World J Surg Oncol 2011; 9:173-173.

18. Innasimuthu AL, Rao GK, Wsong P. Persistent left-sided superior vena cava -a pacing challenge. Acute Card Care 2007; 9:252-252.

19. Dissmann R, Schröder J, Völler H, Behrens S. Entrapment of pacemaker lead by a large net- like Eustachian valve within the right atrium. Clin Res Cardiol 2006; 95(4):241-243.

20. Li T, Xu Q, Liao HT, Asvestas D, Letsas K.P, Li Y. Transvenous dual-chamber pacemaker implantation in patients with persistent left superior vena cava. BMC Cardiovasc Disord 2019; 19(1):1-6.

21. Sasaki K., Tateishi S, Sawada C. Usefulness of a lead delivery system consisting of a fixedshaped sheath and a lumenless bipolar lead in a patient with absent right and persistent left superior vena cava: A case report. Indian Pacing Electrophysiol J 2018;18(6):234-236. 